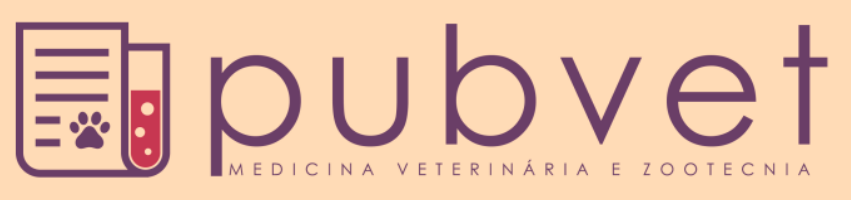

HTTP://DX.DOI.ORG/10.22256/PUBVET.V11N9.882-888

\title{
Hérnia perineal complicada com envolvimento de intestino e bexiga em cão: Relato de caso
}

\author{
Richard Elâino de Oliveira Ferraz ${ }^{1 *}$, Ingrid Rabelo Rodrigues ${ }^{2}$ Hermógenes Josniel \\ Rocha Macedo $^{2}$, Átilla Holanda de Albuquerque ${ }^{3}$, Aline Silveira Feitosa ${ }^{2}$, Victor Manuel \\ de Lacerda Freitas ${ }^{4}$, André Lacerda de Abreu Oliveira ${ }^{5}$ \\ ${ }^{I}$ Médico Veterinário, Prof. - Faculdade Terra Nordeste (FATENE), Hospital Veterinário Metropolitano, setor de clínica médica, Caucaia, \\ Ceará, Brasil. E-mail: richard.ferraz@fatene.edu.br \\ ${ }^{2}$ Graduando(a) em Medicina Veterinária pela Faculdade Terra Nordeste, Campus Caucaia, Ceará, Brasil, E-mail: \\ ingridrabelorodrigues@gmail.com; hjosniel@yahoo.com.br; alinefeitosa12@yahoo.com.br \\ ${ }^{3}$ Doutor em Medicina Veterinária pela Universidade Estadual do Ceará, Campus Itaperi, Ceará, Brasil, E-mail: atillaholanda@hotmail.com \\ ${ }^{4}$ Graduando em Medicina Veterinária pela Universidade Estadual do Ceará, Campus Itaperi, Ceará, Brasil, E-mail: \\ victorlacerdafreitas@gmail.com \\ ${ }^{5}$ Médico Veterinário, DSc. Centro de Ciências e Tecnologias Agropecuárias (CCTA), Universidade Estadual Norte Fluminense Darcy Ribeiro \\ (UENF), Rio de Janeiro, Brasil, E-mail: lacerdavet@uol.com.br \\ *Autor para correspondência, E-mail: richard.fatene@gmail.com
}

RESUMO. A hérnia perineal resulta do enfraquecimento e separação no diafragma pélvico, promovendo deslocamento de órgãos abdominais ou pélvicos. Essa afecção pode ser influenciada por fatores como sexo, hiperplasia e cistos prostáticos, obstipação crônica, dentre outros fatores. O diagnóstico é feito por anamnese, exame físico, ultrassonografia e radiografia. $\mathrm{O}$ objetivo deste trabalho foi relatar o tratamento cirúrgico de hérnia perianal complicada com envolvimento de cólon e bexiga. O conjunto de manobras cirúrgicas mostrou-se uma alternativa para o tratamento de hérnia perianal.

Palavras chave: canino, evisceração perianal, tela cirúrgica

\section{Complicated perineal hernia with bladder and bowel involvement: Case report}

ABSTRACT. Perineal hernia results from weakening and separation in the pelvic diaphragm, promoting displacement of abdominal or pelvic organs. This condition can be influenced by factors such as sex, hyperplasia and prostatic cysts, chronic constipation, among other factors. The diagnosis is made by anamnesis, physical examination, ultrasonography and radiography. The objective of this study was to report the surgical treatment of complicated perianal hernia with involvement of the colon and bladder. The set of surgical maneuvers proved to be an alternative for the treatment of perianal hernia.

Keywords: Canine, perianal evisceration, surgical site

\section{Hernia perineal complicada con comprometimiento del intestino y la vejiga en perro: Reporte de un caso}

RESUMEN. La hernia perineal es resultado del debilitamiento y la separación en el diafragma pélvico, promoviendo el dislocamiento de órganos abdominales o pélvicos. Esta condición puede estar influenciada por factores tales como el género, hiperplasia y quistes prostáticos, estreñimiento crónico, entre otros factores. El diagnóstico se realiza mediante anamnesis, exploración física, ecografía y radiografía. El objetivo de este estudio fue describir el tratamiento quirúrgico de la hernia complicada perianal con afectación del 
colon y la vejiga. El conjunto de maniobras quirúrgicas demostró ser una alternativa para el tratamiento de hernia perianal.

Palabras clave: Canino, evisceración perianal, mallas quirúrgicas

\section{Introdução}

A hérnia perineal caracteriza-se como uma falha dos músculos do diafragma pélvico em manter a sustentação adequada das estruturas pélvicas e/ou abdominais, resultando em uma herniação das mesmas para a região perineal (Tilley et al., 2008). Tendo como fatores predisponentes a atrofia muscular, prostatopatias hiperplásicas, a genética, além de constipação, tenesmo crônico e retopatias recorrente (Bojrab, 2005). A maior prevalência ocorre em caninos machos não castrados com idades e maior incidência entre 7 a 9 anos (Moraes et al., 2013, Ramírez et al., 2015). Os sinais clínicos mais frequentes incluem tumefação da região perineal, tenesmo, disquesia, incontinência urinaria e/ou fecal, estrangúria, anúria, êmese, esta última devido a envolvimento urinário e/ou intestinal (Tilley et al., 2008, Nelson and Couto, 2015).

O diagnóstico pode ser baseado na anamnese, exame físico e exames complementares como radiografia simples ou contrastada e ultrassonografia (Bojrab, 2005, Moraes et al., $\underline{2013}$ ).

O manejo pré-operatório é baseado em enemas, remoção digital das fezes, cateterização urinária e tratamento de suporte (Bojrab, 2005). A herniorrafia pode ser feita com técnicas de transposição anatômica e uso de implantes cirúrgicos biocompatíveis, como o pericárdio bovino (Marques et al., 2015) e malha sintética de polipropileno (Tilley et al., 2008). Quando há envolvimento cístico e colônico, podem ser utilizadas técnicas de suporte como a colonopexia e cistopexia (Risselada et al., 2003, Grand et al., 2013, Arruda et al., 2015, Sharma et al., 2016), também sendo possível realizar a técnica de orquiectomia quando há prostatopatias (Mann et al., 1989, Fossum, 2014).

O objetivo deste trabalho foi relatar o tratamento cirúrgico para a correção de hérnia perineal com a associação das técnicas de herniorrafia com malha sintética de polipropileno, colopexia e cistopexia.

\section{Relato de caso}

Foi atendido um paciente macho, canino, poodle, com sete anos pesando 9,7 kg no Hospital
Veterinário Metropolitano (HVM) na cidade de Caucaia-CE apresentando controle de endoparasitas há três meses, assim como controle de ectoparasitas, não vacinado, alimentação constituída à base de ração comercial extrusada bis in die, água fornecida ad libitum, criado em residência (casa), sem a companhia de outro cão, com acesso supervisionado a rua, além de histórico médico de hemoparasitose.

Durante a consulta, foi relatada a queixa principal, sendo ela um aumento progressivo de volume na região perineal na última semana, sendo ainda relatado durante a anamnese anorexia e apatia que persistiam por uma semana, sendo que no dia anterior o paciente começou a apresentar disquesia, além de hematúria e êmese relatadas no mesmo dia do atendimento (Figura 1).

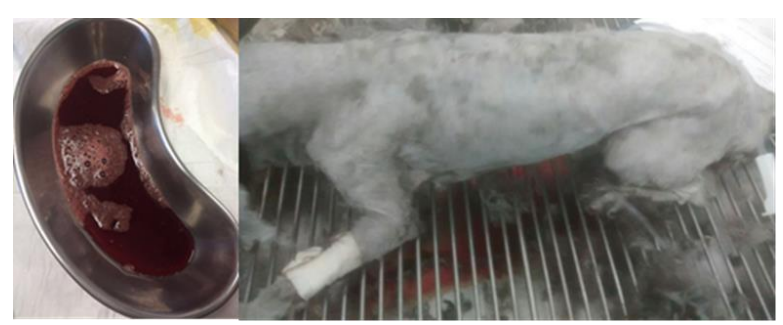

Figura 1. Hematúria observada no período pré-operatório.

No exame físico o mesmo apresentava depressão, linfonodos palpáveis, escore corporal 2, aferido 150 batimentos cardíacos e 56 movimentos respiratórios por minuto, com tempo de perfusão capilar de 5 segundos, pulso fraco e temperatura retal de $38,5^{\circ} \mathrm{C}$.

A palpação do volume encontrado na região perineal lateral direita possuía consistência rígida e maciça, enquanto o outro aumento de volume na região perineal ventral apresentava consistência sugestiva de conteúdo líquido (Figura 2).

Os exames hematológicos revelaram trombocitopenia de $60 \mathrm{mil} / \mathrm{mm}^{3}$, hemácias 3.460 milhões $/ \mathrm{mm}^{3}$, hematócrito $22 \%$ e leucocitose de 22.000, enquanto as provas bioquímicas revelaram uréia de $60 \mathrm{mg} / \mathrm{dL}$, creatinina 2,0 $\mathrm{mg} / \mathrm{dL}$, alanina aminotransferase de $86 \mathrm{mg} / \mathrm{dL}$. No exame radiográfico em projeção dorsoventral e latero lateral, foi observado uma massa radiopaca na região perianal (Figura 3).

Diante dos dados de anamnese, exame físico e exames complementares, o paciente foi 
diagnosticado com hérnia perineal e encaminhado para os cuidados pré-operatórios (Figura 4).

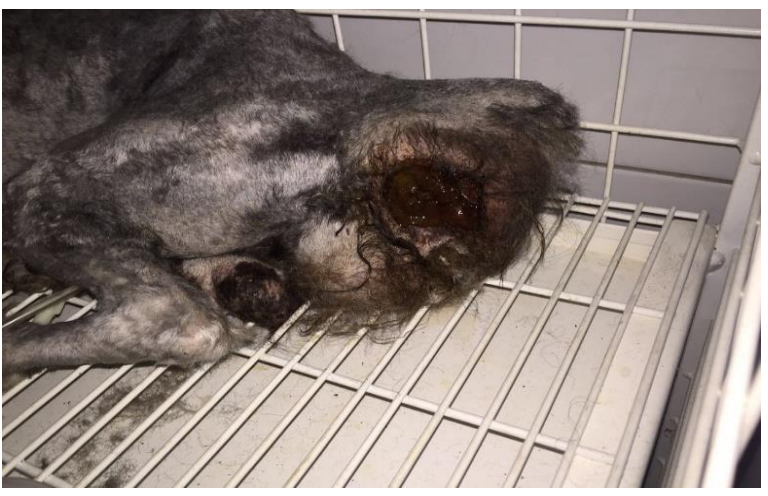

Figura 2. Imagem demonstrando a presença de uma massa na região perineal.

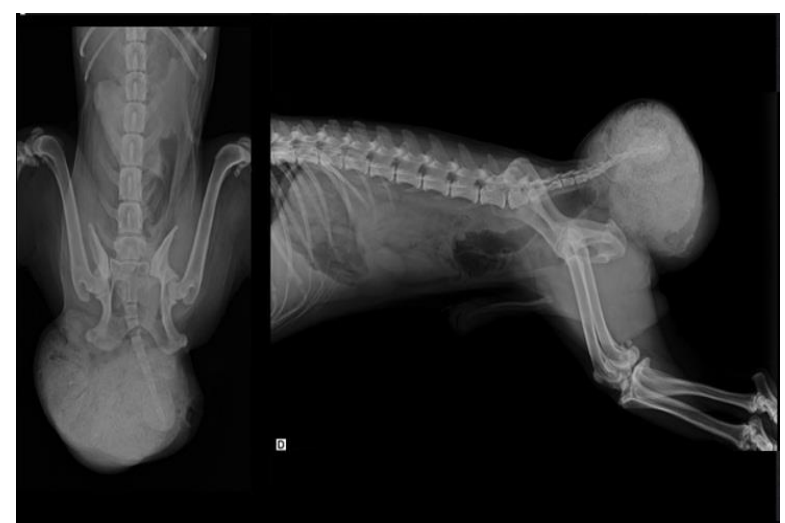

Figura 3. Imagem radiográfica em projeção ventri-dorsal e latero-lateral demonstrando um imagem radiopaca de contornos arredondados em região mais ventral (A) e outra com radiopacidade bastante aumentada em região mais dorsal (B).

Nos cuidados pré-operatórios, o paciente foi submetido à fluidoterapia a base de ringer com lactato por via intravenosa, além de iniciado um protocolo antimicrobiano a base de ceftriaxona $30 \mathrm{mg} / \mathrm{kg}$ associado a metronidazol $15 \mathrm{mg} / \mathrm{kg}$ ambos a cada 12 horas. Diante do quadro de dor apresentado pelo paciente, este teve que ser submetido a anestesia epidural com lidocaína, para realização do exame de palpação digital do reto onde foi observado presença de fezes endurecidas e ressecadas na ampola retal e posteriormente realizado enema e remoção manual das fezes.

Devido aos sinais clínicos indicativos de envolvimento urinário, foi eleito inicialmente o procedimento de cateterismo vesical, sem sucesso, provavelmente devido à obstrução urinária causada pela grande quantidade de fezes na massa herniada. Mesmo após a remoção manual das fezes, não houve sucesso no procedimento supra citado, sendo assim, foi eleita uma segunda medida para descompressão da bexiga, onde foi então feita punção da mesma no conteúdo herniado.

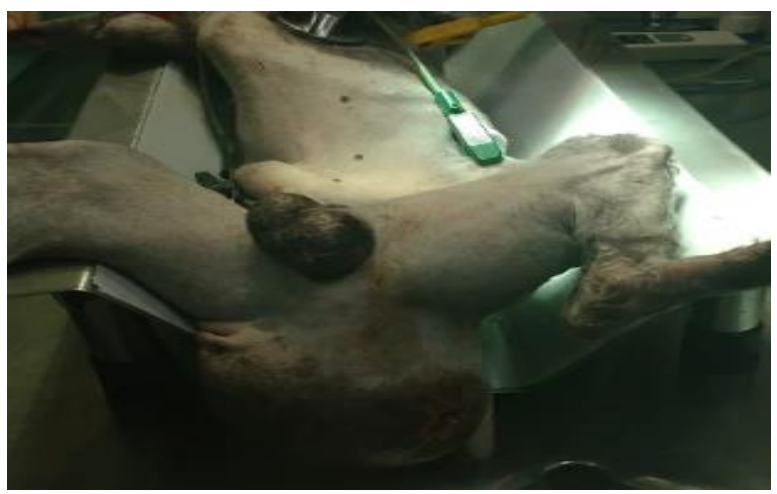

Figura 4. Pré-operatório do paciente em decúbito dorsal, onde pode ser notada diminuição no conteúdo herniado, após esvaziamento da ampola retal e punção da bexiga.

$\mathrm{Na}$ medicação pré-anestésica utilizou-se cloridrato de acepromazina na dose de $0,05 \mathrm{mg} / \mathrm{kg}$ e cloridrato de tramadol na dose de 2,0 mg/ $\mathrm{kg}$, ambos por via intravenosa. Como indução anestésica utilizou-se diazepam na dose de 0,5 $\mathrm{mg} / \mathrm{kg}$, seguido de cloridrato de cetamina, $5 \mathrm{mg} / \mathrm{kg}$ ambas por via intravenosa e manutenção anestésica com gás isoflurano.

Foi realizado uma incisão semi circular na região da hérnia, sendo que a perineotomia revelou o anel herniário com aderência do omento à musculatura circunjacente e presença de alças jejunais (Figura 5). A aderência do omento foi desfeita através de divulsão romba, seguida do reposicionamento manual do omento e alças jejunais na cavidade abdominal.

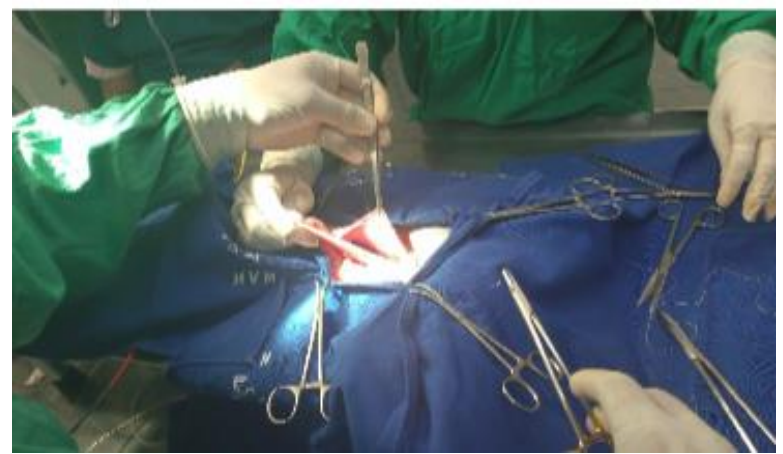

Figura 5. Aderência do omento em musculatura pélvica e alças intestinais compondo o conteúdo herniado

Após a redução da hérnia, foi colocada uma tela de polipropileno utilizando fio de sutura de Nylon diâmetro 2-0 em padrão simples separado. Por último foi realizado o fechamento de pele como de rotina e realizada a castração (ㅌgura 6).

Foi realizada celiotomia mediana xifo púbica (Figura 7), seguida de fixação do cólon na parede 
abdominal (Figura 8) com sutura contínua simples transfixando a serosa previamente escarificada com a musculatura abdominal, na qual também foi previamente realizada uma incisão para uma posterior aderência. Foi observado que a bexiga urinária havia retornado a cavidade abdominal, provavelmente após a cistocentese da mesma.

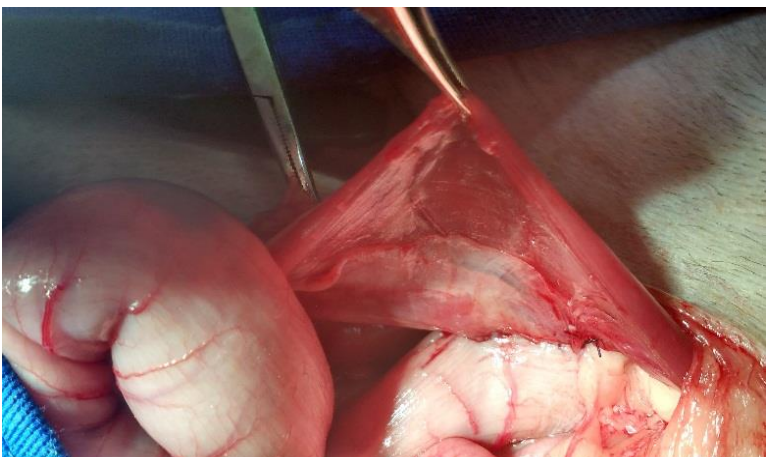

Figura 6. Paciente no momento da herniorrafia em decúbito esternal, sendo a técnica realizada com implante de polipropileno fixada com padrão de sutura simples separado, para correção de hérnia perineal.

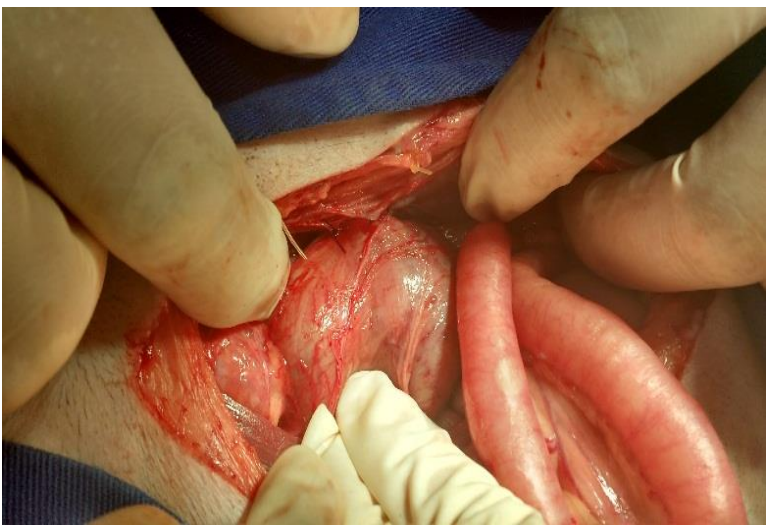

Figura 7. Incisão pré-retro-umbilical para realização de celiotomia exploratória.

No procedimento de cistopexia realizou-se também escarificação prévia, seguida de fixação com padrão de sutura simples contínuo com fio de poliglactina 910 (Figura 9). Ainda durante a celiotomia exploratória foi observado hiperplasia prostática (Figura 10).

A miorrafia do abdome foi realizada com fio nylon 2-0 e padrão de sutura Reverdin, seguido de uma segunda camada de sutura com padrão Cushing e o fechamento da pele com Nylon 2-0 e padrão de sutura simples separado.

No pós-operatório imediato foi administrado ao paciente tramadol $2 \mathrm{mg} / \mathrm{kg}$ a cada 8 horas e cetoprofeno na dose de $1 \mathrm{mg} / \mathrm{kg}$ a cada 24 horas, além de continuidade de fluidoterapia e protocolo antimicrobiano iniciado no pré-operatório. O animal retornou com 14 dias para retirada de pontos, onde foi reavaliado. Suas funções de micção e defecação haviam retornado ao normal. O mesmo não apresentou quadro de recidivas nos seis primeiros meses após a cirurgia (Figura 11)

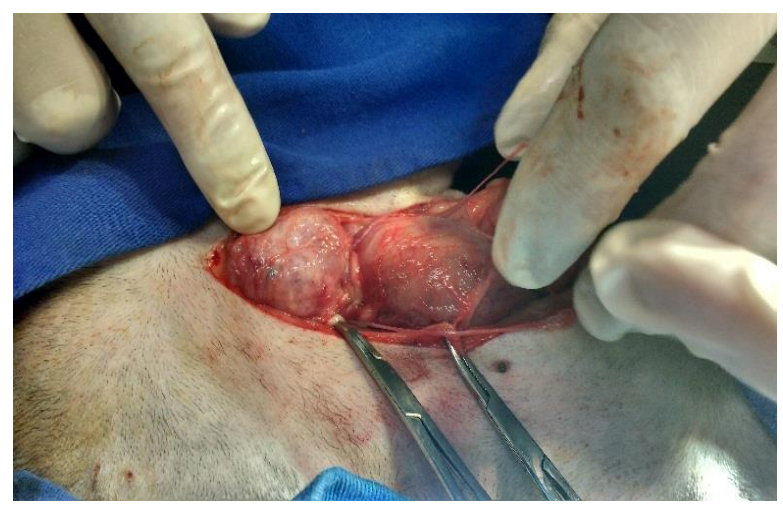

Figura 8. Imagem do acabamento final de colopexia em parede abdominal, com sutura simples contínua, transfixando a serosa com fio poliglactina $9103-0$

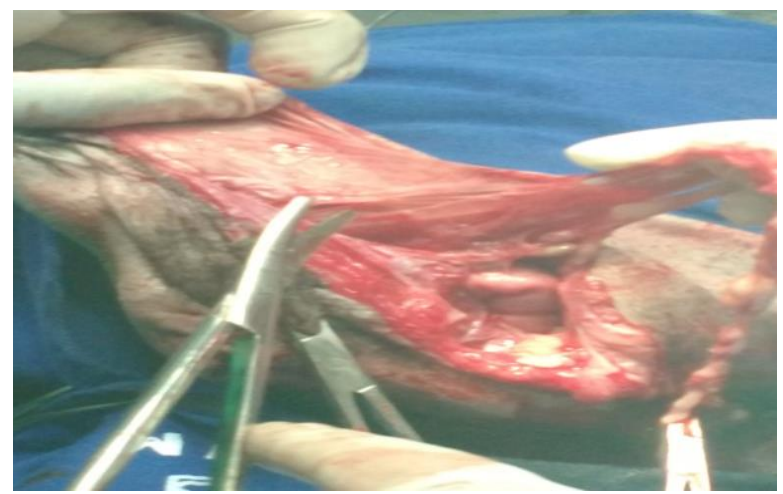

Figura 9. Imagem do acabamento final da cistopexia em parede abdominal, com padrão de sutura simples contínuo utilizando fio de poliglactina 910 .

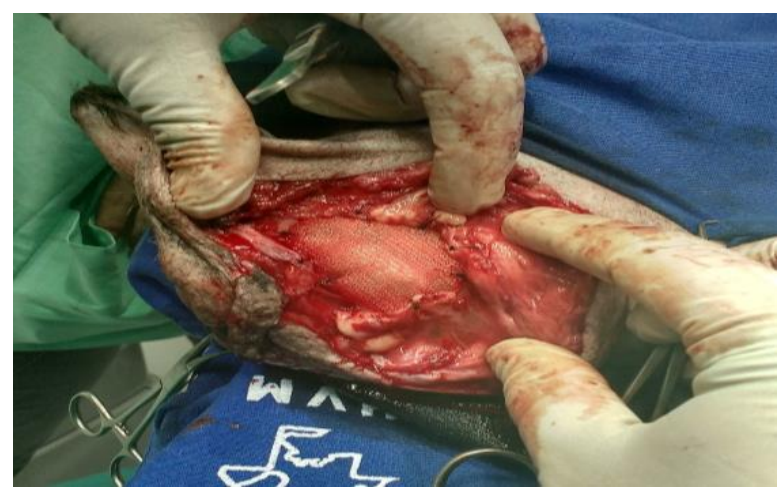

Figura 10. Próstata hiperplásica durante a inspeção da cavidade pélvica.

\section{Discussão}

No exame físico o paciente apresentou desconforto à palpação, possivelmente devido ao comprometimento vesical, uma vez que a tumefação perineal geralmente é dolorosa quando há envolvimento vesical (Bojrab, 2005). Nos exames complementares observou-se anemia, 
hematócrito baixo, trombocitopenia, leucocitose, aumento de ureia, creatinina e alanina aminotransferase, sendo que a descrição desses achados laboratoriais não são comumente relatados por outros autores. No entanto, relatouse azotemia pós-renal secundaria a obstrução urinária (Tilley et al., 2008). Após anestesia peridural com objetivo de diminuir a contaminação no transoperatório foi realizado enema e remoção manual das fezes no reto durante o pré-operatório imediato, conforme preconizado em literatura em que Costa Neto et al. (2006) realizaram enema num período de 60 minutos antes da cirurgias de herniorrafia.

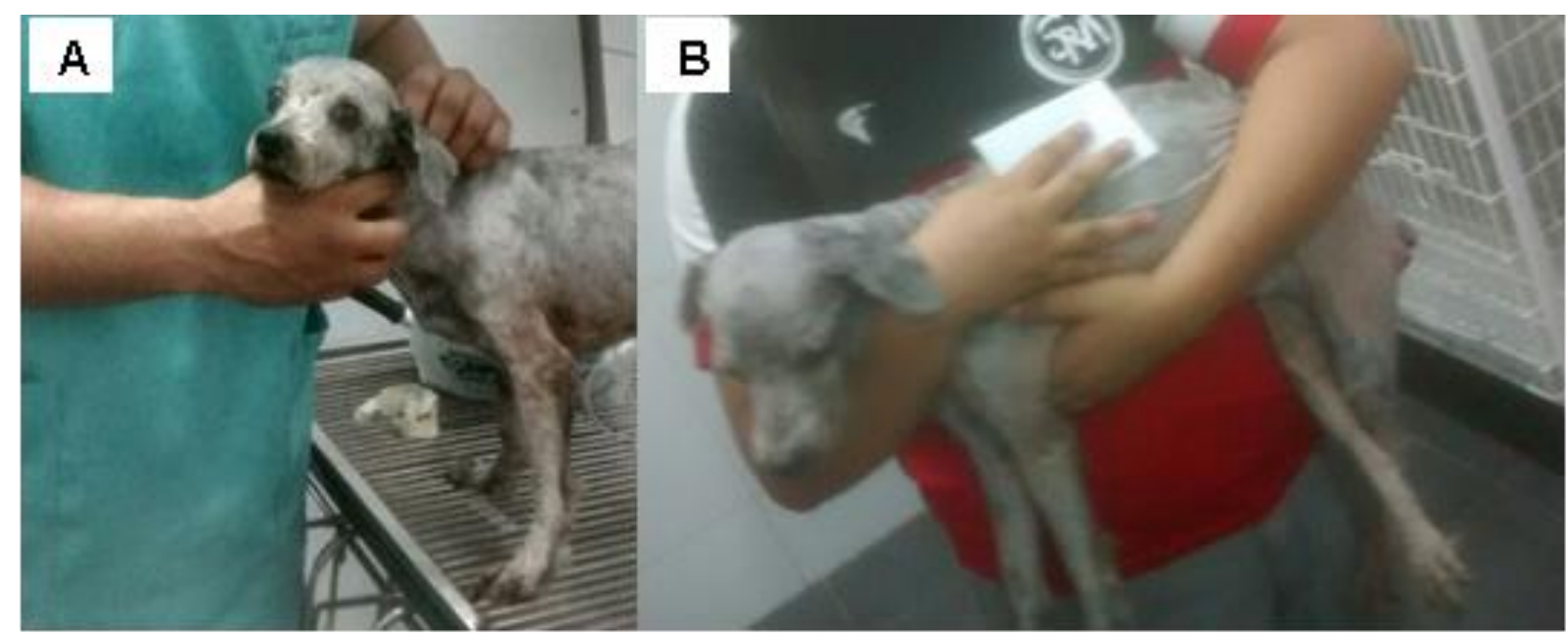

Figura 11. Animal no pós-operatório imediato (A), e seis meses após a cirurgia (B).

A confirmação do envolvimento urinário é feita por exame ultrassonográfico, cateterização da bexiga urinária ou punção da massa herniada (Nelson and Couto, 2015). Diante do quadro de hematúria e incontinência urinaria, foi eleito inicialmente o procedimento de cateterismo vesical, sem sucesso, provavelmente devido à obstrução urinária causada pela grande quantidade de fezes na massa herniada. Foi então feita punção de alívio para descompressão da bexiga, confirmando seu envolvimento.

No caso deste relato foi adotado o uso da técnica de implante biocompatível para a herniorrafia, devido este demonstrar menores taxas de recidivas (Bellenger, 1980, Marques et al., 2015, Shaughnessy and Monnet, 2015), embora existam relatos de outras técnicas combinadas como a elevação do músculo obturador interno, colocação de tela de polipropileno e transposição do músculo glúteo superficial em casos de recidivas (Argüelles et al., 2004).

No presente caso a herniorrafia foi realizada com implante de polipropileno; porém outros implantes mais baratos e menos acessíveis possuem a mesma eficácia e são indicados na literatura, dentre eles estão o pericárdio e peritônio bovino conservados em glicerina, não sendo relatados com rejeição (Bellenger, 1980, Matera et al., 1981, Daleck et al., 1992, Marques et al., 2015,
Villamil and Carrera, 2016). Todavia, em estudos quando utilizando a técnica de herniorrafia com implantes de pericárdio equino conservado em glicerina, observou que todos os cães submetidos ao procedimento apresentaram processo inflamatório e fibrose discreta (López et al., 2007).

O envolvimento cístico e do colón em hérnias perineais já foi relatado por outros autores, adotando a cistopexia e colopexia como medidas profiláticas afim de evitar quadro recidivo ou herniação contralateral (Risselada et al., 2003, Grand et al., 2013, Arruda et al., 2015, Sharma et al., 2016). Os achados de hiperplasia prostática, além do tenesmo no paciente, isoladamente não são considerados causas suficientes desta afecção; porém a união dos dois fatores pode causar uma força caudal contra os músculos do diafragma pélvico, favorecendo a formação de uma hérnia perineal (Bojrab, 2005, Brissot et al., 2004, Rai and Chandrapuria, 2016).

Após o tratamento cirúrgico de herniorrafia foi adotada a orquiectomia, visto que cães submetidos a esta técnica apresentaram uma taxa 2,7 vezes menor de recidivas (Tilley et al., 2008), embora autores como Mann et al. (1989) afirmam que a orquiectomia não deve ser recomendada em associação com o tratamento de hérnia perineal, a menos que o paciente apresente alguma complicação prostática, de acordo com o caso do paciente relatado. 
No pós-operatório o paciente teve uma evolução boa, apresentando quadro de incontinência urinária apenas nas primeiras 12 horas, as fezes voltaram a consistência normal após 48 horas, diferente do prescrito na literatura que afirma a possibilidade de complicações com recidiva herniária, infecção com formação de abscessos e fístulas reto cutâneas, tenesmo, prolapso retal, incontinência fecal e ou urinária, lesão do nervo ciático, predispondo uma taxa de mortalidade de $30 \%$ entre os cães com vesícula urinária retrofletida (Arruda et al., 2015, Shaughnessy and Monnet, 2015).

\section{Conclusão}

A técnica de cistopexia, colopexia, associadas à colocação de tela de polipropileno mostrou-se uma alternativa para tratamento de hérnia perianal com envolvimento de bexiga e cólon e fecaloma em canino.

\section{Agradecimentos}

Agradecemos ao Hospital Veterinário Metropolitano de Caucaia (HVM) e a Faculdade Terra Nordeste sede Caucaia (FATENE) juntamente com a Coordenação do Curso de Medicina Veterinária da FATENE com apoio técnico e incentivo.

\section{Referências Bibliográficas}

Argüelles, J. C., Ishimi, C. \& Baquedano, M. 2004. Hernia perineal en el perro. Combinación de tres técnicas para la corrección quirúrgica. Tres casos clínicos. Clínica Veterinaria de Pequeños Animales, 24, 161-166.

Arruda, M. M. L., Carneiro, L. Z., Waschburger, D. J., Soares, B., Pes, B., Grundemann, J. \& Mallmann, L. 2015. Cistopexia por sondagem pré-púbica no tratamento de retroflexão vesical redicivante em hérnia perineal em cão. Relato de caso. Acta Veterinaria Brasilica, 8, 226230.

Bellenger, C. R. 1980. Perineal hernia in dogs. Australian Veterinary Journal, 56, 434-438.

Bojrab, M. J. 2005. Técnicas atuais em cirurgia de pequenos animais. Editora Roca, São Paulo.

Brissot, H. N., Dupré, G. P. \& Bouvy, B. M. 2004. Use of laparotomy in a staged approach for resolution of bilateral or complicated perineal hernia in 41 dogs. Veterinary Surgery, 33, 412421.
Costa Neto, J. M., Menezes, V. P., Toríbio, J. M. d. M. L., Oliveira, E. C. S., Anunciação, M. C., Teixeira, R. G., D'Assis, M. J. M. H. \& Vieira Júnior, A. S. 2006. Tratamento cirúrgico para correção de hérnia perineal em cão com saculação retal coexistente. Revista Brasileira de Saúde de Produção Animal, 7, 7-19.

Daleck, C. R., Padilha Filho, J. G., Daleck, C. L. M. \& Costa Neto, J. M. 1992. Reparação de hérnia perineal em cães com peritônio de bovino conservado em glicerina. Ciência Rural, 22, 179-183.

Fossum, T. W. 2014. Cirurgia de pequenos animais, 4 edn. Elsevier Brasil, São Paulo.

Grand, J.-G., Bureau, S. \& Monnet, E. 2013. Effects of urinary bladder retroflexion and surgical technique on postoperative complication rates and long-term outcome in dogs with perineal hernia: 41 cases (20022009). Journal of the American Veterinary Medical Association, 243, 1442-1447.

López, J., Guaimás, L., Báez, A., Lockett, M. \& Maidana, R. 2007. Tratamiento quirúrgico de hernias perineales en caninos mediante el uso de pericardio equino conservado en glicerina. Revista Veterinaria, 18, 3-8.

Mann, F. A., Boothe, H. W., Amoss, M. S., Tangner, C. H., Puglisi, T. A. \& Hobson, H. P. 1989. Serum testosterone and estradiol 17-beta concentrations in 15 dogs with perineal hernia. Journal of the American Veterinary Medical Association, 194, 1578-1580.

Marques, D. R. C., Russo, C. \& Ibañez, J. F. 2015. Utilização de pericárdio bovino conservado em glicerina $98 \%$ na herniorrafia perineal em cães-Relato de 12 casos. Arquivos de Ciências Veterinárias e Zoologia da UNIPAR, 18, 185190.

Matera, A., Moraes Barros, P. S., Stopiglia, A. J. \& Randi, R. E. 1981. Hérnia perineal no cão. Tratamento cirúrgico mediante utilização de malha de polipropileno. Revista da Faculdade de Medicina Veterinária e Zootecnia da Universidade de São Paulo, 18, 37-41.

Moraes, P. C., Zanetti, N. M., Burger, C. P., Meirelles, A. E. W. B., Canola, J. C. \& Isola, J. G. M. P. 2013. Correction of rectal sacculation through lateral resection in dogs with perineal hernia-technique description. Arquivo Brasileiro de Medicina Veterinária e Zootecnia, 65, 654-658. 
Nelson, R. W. \& Couto, C. G. 2015. Medicina interna de pequenos animais. Elsevier Editora, Amsterdan.

Rai, S. \& Chandrapuria, V. P. 2016. Surgical management of perineal hernia with prostatic hyperplasia. Intas Polivet, 17, 153-154.

Ramírez, A., Pastor, N., Durán, M. E., Gutiérrez, A. \& Ezquerra, L. J. 2015. Hernia perineal en el perro, un estudio de prevalencia de 81 casos. Archivos de Medicina Veterinaria, 47, 71-75.

Risselada, M., Kramer, M., Velde, B., Poils, I. \& Görtz, K. 2003. Retroflexion of the urinary bladder associated with a perineal hernia in a female cat. Journal of Small Animal Practice, 44, 508-510.

Sharma, A. K., Kumari Chandrakala, L. K., Singh, S., Kumar, S. \& Kumar, P. 2016. Successful surgical management of recurrent perineal hernia using colopexy and cystopexy in a dog. International Journal of Livestock Research, 6, 105-109.
Shaughnessy, M. \& Monnet, E. 2015. Internal obturator muscle transposition for treatment of perineal hernia in dogs: 34 cases (1998-2012). Journal of the American Veterinary Medical Association, 246, 321-326.

Tilley, L. P., Smith, J. R. \& Francis, W. K. 2008. Consulta veterinária em 5 minutos: Espécies canina e felina. Editora Manole, São Paulo.

Villamil, C. S. \& Carrera, A. E. 2016. Perineal herniorrhaphy in a dog using a cone-shaped polypropylene mesh implant. Veterinary Record Case Reports, 4, e000298.

\section{Article History:}

Received 28 April 2017

Accepted 25 May 2017

Available online 7 August 2017

License information: This is an open-access article distributed under the terms of the Creative Commons Attribution License 4.0, which permits unrestricted use, distribution, and reproduction in any medium, provided the original work is properly cited 